\title{
中国脊椎动物2020年新增物种
}

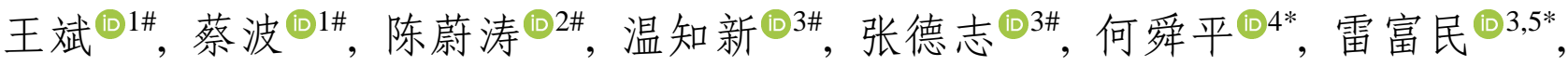 \\ 杨奇森 (1) , 江建平(1*
}

1. 中国科学院成都生物研究所, 成都 610041; 2. 中国水产科学研究院珠江水产研究所, 广州 510380; 3. 中国科学院动物研究所, 北京 100101; 4. 中国科学院水生生物研究所, 武汉 430072; 5. 中国科学院大学, 北京 100049

\begin{abstract}
摘要: 为了及时掌握中国脊椎动物新增物种情况，本文系统检索和整理了2020年发表的分类学文献，汇总结果表明：2020年 中国脊椎动物共新增 109 种, 其中新物种 100 种, 国家级新记录种 9 种。包括鱼类新种 24 种、两栖类新种 41 种和新记录 4 种、爬 行类新种 30 种和新记录 4 种、鸟类新种 1 种、哺乳类新种 4 种和新记录 1 种。上述新增物种中有 92 种描述报道时应用了分子遗传 学证据, 占新增物种数量的 $84.4 \%$ 。在新增脊椎动物物种中, 两栖类集中于无尾目、爬行类全部属于有鳞目, 分别有 43 种和 34 种, 其累计占比超新增物种总数的 $70 \%$ 。在云南、西藏、湖南、贵州、四川等省区发现新物种数量较多, 均有 10 种及以上，累 计占比超新增物种总数的 $60 \%$ 。绝大部分物种为中国学者发表, 绝大多数论文发表于英文期刊。总结数据提示今后需持续加 强我国低等脊椎动物多样性的调查研究, 重视运用分子系统学技术进行物种识别。
\end{abstract}

关键词: 脊椎动物; 新物种; 新记录; 中国

王斌, 蔡波, 陈蔚涛, 温知新, 张德志, 何舜平, 雷富民, 杨奇森, 江建平 (2021) 中国脊椎动物2020年新增物种. 生物多样性, 29, 1021-1025. doi: 10.17520/biods.2021214.

Wang B, Cai B, Chen WT, Wen ZX, Zhang DZ, He SP, Lei FM, Yang QS, Jiang JP (2021) New vertebrate forms discovered in China in 2020. Biodiversity Science, 29, 1021-1025. doi: 10.17520/biods.2021214.

\section{New vertebrate forms discovered in China in 2020}

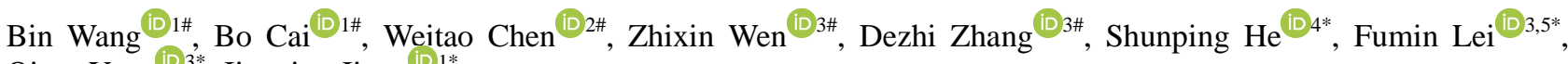
Qisen Yang ${ }^{\left(3^{*}\right.}$, Jianping Jiang ${ }^{\mathbb{( \mathbb { } 1 *}}$

1 Chengdu Institute of Biology, Chinese Academy of Sciences, Chengdu 610041

2 Pearl River Fisheries Research Institute, Chinese Academy of Fishery Sciences, Guangzhou 510380

3 Institute of Zoology, Chinese Academy of Sciences, Beijing 100101

4 Institute of Hydrobiology, Chinese Academy of Sciences, Wuhan 430072

5 University of Chinese Academy of Sciences, Beijing 100049

\section{ABSTRACT}

Aim: To review the new species and new national records of vertebrates discovered in China in 2020.

Methods: We compiled and analyzed the taxonomic literature on China's fauna published in 2020.

Results: In 2020, 100 new species and nine new national records of vertebrates were discovered in China. These include: 24 new species of fishes; 41 new species and four new national records of amphibians; 30 new species and four new national records of reptiles; one new species of bird; and four new species and one new national record of mammals. Of these newly recorded vertebrate species, molecular genetic data were used to evaluate 92 of the species described, which constituted $84.4 \%$ of the total number of newly recorded species. Most of these species are within the classes Amphibia and Reptilia; the orders Anura and Squamata constituted $70 \%$ of the total newly recorded vertebrate species. Geographically, more than $60 \%$ of the newly recorded vertebrate species were discovered in southwestern China; Yunnan, Xizang, Hunan, Guizhou, and Sichuan provinces each had 10 or more new species described in 2020.

收稿日期: 2021-05-25; 接受日期: 2021-08-01

基金项目: 中国科学院战略性先导科技专项地球大数据科学工程项目(XDA19050201)、第二次青藏高原综合科学考察项目 (2019QZKK0501; 2019QZKK0402)和中国生物多样性监测与研究网络(Sino BON)

\# 共同第一作者 Co-first authors

* 共同通讯作者 Co-authors for correspondence. E-mail: Jiangjp@cib.ac.cn; clad@ihb.ac.cn; leifm@ioz.ac.cn; yangqs@ioz.ac.cn 
The majority of the species were described by Chinese researchers and most of their findings were published in English language journals.

Conclusion: These results indicate that taxonomic studies, particularly those of the basal groups of vertebrates, should be the continuous focus of biodiversity research in China. Future studies should adopt an integrative taxonomic approach and include molecular genetic data.

Key words: vertebrates; new species; new record; China

复杂多样的地质历史、气候和生态环境乃育了 我国丰富而独特的生物多样性。在我国生物分类学 家长期的艰苦奋斗下, 《中国植物志》和《中国动 物志》相继问世, 标志着我国的动植物本底基本被 摸清。然而, 志书编著周期长, 其数据存在较高的 局限性。据不完全统计, 中国已知有哺乳类693种 (蒋志刚等, 2017), 鸟类1,445种(郑光美, 2017), 爬 行类511种(王剀等, 2020), 两栖类514种(王剀等, 2020), 鱼类4,949种(The Biodiversity Committee of Chinese Academy of Sciences, 2021)。且近期又有大 量的新物种被发现和描述 (The Biodiversity Committee of Chinese Academy of Sciences, 2021)。

生物物种名录是一个自然地理区域或行政单 元的生物区系本底资料, 它的及时更新对于生物多 样性保护研究、管理和监测具有重要的指导意义(马 克平, 2015)。因此, 及时总结和编制生物物种名录 并建立及时更新制度, 可为中国脊椎动物分类和保 护等相关工作提供数据支撑。本文系统检索和整理 了2020年发表的分类学文献(附录1), 并通过查阅相 关网站, 如Amphibian Species of the World (Frost, 2021)、The Reptile Database (Uetz et al, 2021)和IOC 世界鸟类名录(Gill et al, 2021), 汇总了 2020年度 (2020年1月1日至2020年12月31日)中国境内脊椎动 物新物种和国家新记录物种。

\section{新发表物种的基本状况}

2020年中国脊椎动物新增109种, 其中新种 100 种(含3亚种提升为种), 国家新记录9种(附录1)。新 增脊椎动物物种中, 鱼类新种 24 种, 两栖类新种 41 种、国家新记录 4 种, 爬行类新种 30 种、国家新记录 4 种, 鸟类新种 1 种 (亚种提升为种), 哺乳类新种 4 种 (含2亚种提升为种)、国家新记录1种。两栖类和爬 行类新增物种数分别占 $41.3 \%$ 和 $31.2 \%$, 这两个类群 总体占比超过 $70 \%$; 鱼类占 $22.0 \%$; 而哺乳类和鸟 类只分别占 $4.6 \%$ 和 $0.9 \%$ 。这提示鱼类、两栖类和爬
行类仍有大量未知种尚待发现或描述, 需要持续加 大调查研究力度, 以便尽早认知这些类群的物种多 样性状况。鸟类和哺乳类的新增物种很少, 远远低 于其他脊椎动物类群, 这表明它们的区系分类调查 研究工作可能相对比较系统全面。

2020年中国新增的109种脊椎动物中, 92种的 发表文献应用了详细的分子系统学证据, 占新增物 种总数的 $84.4 \%$ 。其中鱼类仅有 7 种应用了分子证据, 只占 24 种新增鱼类物种的 $29.2 \%$, 而其他 4 个类群的 新增物种的发表全部都采用了分子证据。在发表的 新增物种中, 线粒体基因组的16S rRNA、COI、Cyt $b$ 、 $N D 2 、 N D 4$ 等基因(片段)的DNA序列最常用于构建 系统发育树。这提示分子系统学证据已成为物种识 别的重要依据。

\section{新增物种归录的目科属}

2020年中国109种新增脊椎动物物种隶属13目 31 科51属。

在所涉及的 13 目中, 两栖类的无尾目新增物种 最多, 达到 43 种, 占比 $39.4 \%$; 其次是爬行类的有鳞 目新增 34 种, 占比 $31.2 \%$; 接下来是鱼类的鲤形目 新增9种(占比 $8.3 \%$ )、鲇形目和鲇形目各6种(各占 $5.5 \%$ ); 其他目都少于 3 种，占比少于 $3 \%$ (附录2)。表 明这些新增物种主要集中于低等脊椎动物类群中 物种多样性最丰富的几个目: 两栖类中的无尾目物 种数在整个两栖类中的占比超过 $80 \%$, 爬行类中的 有鳞目物种数在整个爬行类中的占比超过 $90 \%$, 鱼 类中的鲇形目和鲤形目均是鱼类里的绝对优势目。

在所涉及的 31 科中, 两栖类的角蟾科和蛙科新 增物种最多, 分别为 23 种和 14 种, 占比分别为 $21.1 \%$ 和 $12.8 \%$; 其次是爬行类的镾蚚科有 8 种, 占 $7.3 \%$; 接下来是鲤科、树蛙科和游蛇科各6种(各占 $5.5 \%$ ), 鮡科5种(占 $4.6 \%$ ); 其他科少于 5 种(附录2)。 从科级水平上来看, 新增物种也主要集中于物种多 样性高的科。角蟾科和蛙科是中国两栖类物种数量 
最多的科, 而游蛇科则是爬行类中物种数量最多的 科; 鲤科和鮡科是中国常见的鱼类科。

在所有 51 属中, 两栖类角蟾科的角蟾属 (Megophrys)和掌突蟾属(Leptobrachella)分别达 12 种和 9 种, 占脊椎动物 2020 年新增物种数的 $11.0 \%$ 和 8.3\%; 爬行类的龙蚚属(Diploderma)、两栖类的湍蛙 属(Amolops)和琴蛙属(Nidirana)分别有7种、7种和5 种, 分别占 $6.4 \% 、 6.4 \%$ 和 $4.6 \%$; 而其他属小于 5 种, 占比小(附录2)。同上, 新增物种数量最多的属也集 中于两栖类和爬行类。

以上结果表明物种多样性水平高的分类单元 乃是新增物种最多的类群, 提示这些类群仍是分类 学的研究热点, 它们的物种多样性水平被明显低估。

\section{新增物种的分布}

109 个新增物种中, 除 9 个国家新记录种外, 已 知黄连山灌树蛙(Raorchestes huanglianshan)明确在 其他国家有分布, 黄连山掌突蟾(Leptobrachella aspera)、滇西琴蛙(Nidirana occidentalis)、伯仲钝头 蛇 (Pareas geminatus) 和沃氏过树蛇 (Dendrelaphis vogeli)尚不能确定在其他国家有分布; 其余的95新 种均为中国特有种，比例达到 $87.1 \%$ 。

109 个新增物种中, 分布于南方 (以祁连山-秦 岭-淮河为界)的物种达到 105 种, 占比 $96.3 \%$, 北方 只有 4 种，只占 $3.7 \%$ 。以七大行政地理分区来看，西 南地区分布了 67 种, 华东地区有23种, 华中地区和 华南地区分别分布了16种和19种，西北地区2种，东 北地区1种, 华北地区没有新增物种分布(图1)。这表 明只有中国陆地面积 $1 / 4$ 的西南地区在2020年新增物 种数量中占比达 $61.5 \%$, 超过其他地理区的总和。

109 个新增物种中, 2 个物种的分布范围跨 4 省, 2 个物种的分布范围跨3省, 8 个物种的分布范围跨 2 省, 而其他 97 个种的分布范围仅涵盖 1 省, 而且很 多物种仅在某个县甚至某个保护区内有分布。许多 新发现的物种分布区非常狭窄, 尤其是两栖动物, 仅分布于某个小区域, 多年调查才被发现, 如角蟾 科的掌突蟾属物种和蛙科的湍蛙属物种。这暗示这 些类群的扩散能力差或狭窄的本地适应性。

在中国34个省级行政区中, 20 个有新增物种分 布、14个未发现分布有新增物种(图1)。新增物种数 量最多的省是云南(新种26种和国家新记录4种), 其 他依次是西藏(新种14种)、湖南(新种13种)、贵州(新

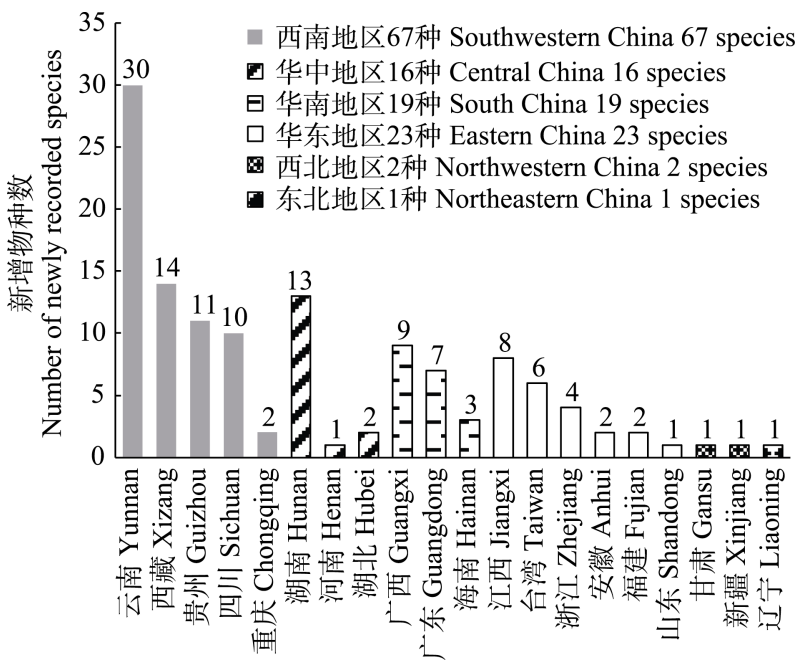

图12020年发表的中国脊椎动物新增物种分布于各行政大 区和各省的数量

Fig. 1 Number of newly recorded vertebrate species in 2020 in major administrative region and provincial unit

种11种)、四川(新种10种)、广西(新种7种和国家新 记录2种)、江西(新种 8 种)和广东(新种6种和国家新 记录1种), 台湾也分布有 6 个新种, 而其他省份分布 新增物种数低于 5 个。从水系看, 2020年长江和珠江 共发现鱼类新种16种, 占我国淡水鱼类新种总数的 $80 \%$ 。这些区域新增物种较多与近年来的深入调查 研究密不可分, 特别是第二次青藏高原综合科学考 察研究的深入开展和生态环境部推进的生物多样 性优先区的多样性调查工作。

此外, 9种国家新记录物种中的6种是在云南或 广西边境地区发现的。这些结果表明在我国南部边 境地区的物种多样性调查研究函待加强, 尤其是有 必要开展跨境物种多样性调查。

\section{发表新增物种的学者及单位}

在 2020年发表的中国脊椎动物 109 个新增物 种中, 共有 126 家单位参与, 其中国外机构 39 家 (附录3); 共有 262 位学者参与, 其中外国学者 38 位(附录4)。

在参与发表新增物种的126家单位中, 参与发 表2-4种的单位有 41家, 5-9种的单位有16家，10个 及以上物种的单位有 4 家(中国科学院成都生物研究 所、中国科学院昆明动物研究所、中山大学和中国 科学院大学) (附录3)。

在参与发表新增物种的262位学者中, 参与发 表2-4种的学者有63位，5-9种的有28位(附录4)；10 
表1 2020年参与发表10个及以上中国新增脊椎动物物种的学者统计

Table 1 Researchers who authored 10 or more newly recorded vertebrate species of China in 2020

\begin{tabular}{|c|c|c|}
\hline 作者 Author & 作者现所属单位 Affiliation & 发表物种数 No. of species published \\
\hline 车静 Jing Che & $\begin{array}{l}\text { 中国科学院昆明动物研究所 Kunming Institute of Zoology, Chinese Academy } \\
\text { of Sciences }\end{array}$ & 23 \\
\hline 蒋珂 Ke Jiang & $\begin{array}{l}\text { 中国科学院成都生物研究所 Chengdu Institute of Biology, Chinese Academy } \\
\text { of Sciences }\end{array}$ & 23 \\
\hline 王英永 Yingyong Wang & 中山大学 Sun Yat-sen University & 17 \\
\hline 吕植桐 Zhitong Lü & 中山大学 Sun Yat-sen University & 17 \\
\hline 王剀 Kai Wang & 俄克拉荷马大学 University of Oklahoma & 15 \\
\hline 王斌 Bin Wang & $\begin{array}{l}\text { 中国科学院成都生物研究所 Chengdu Institute of Biology, Chinese Academy } \\
\text { of Sciences }\end{array}$ & 12 \\
\hline 王健 Jian Wang & 中山大学 Sun Yat-sen University & 12 \\
\hline 颜芳 Fang Yan & $\begin{array}{l}\text { 中国科学院昆明动物研究所 Kunming Institute of Zoology, Chinese Academy } \\
\text { of Sciences }\end{array}$ & 11 \\
\hline 任金龙 Jinlong Ren & $\begin{array}{l}\text { 中国科学院成都生物研究所 Chengdu Institute of Biology, Chinese Academy } \\
\text { of Sciences }\end{array}$ & 11 \\
\hline 曾昭驰 Zhaochi Zeng & 中山大学 Sun Yat-sen University & 10 \\
\hline
\end{tabular}

种以上的有10位，他们来自4家单位(表1)。

上述统计结果表明, 中国学者和有关科研院所 及大专院校在积极广泛地开展中国脊椎动物多样 性的调查研究, 但存在明显的不均衡现状。

\section{5 发表新增物种的出版物}

2020年发表中国新增脊椎动物物种名称的出 版物主要有期刊和专著两种形式(附录1)。其中发表 在专著中的物种有 17 种, 分别发表在《西藏两栖爬 行动物: 多样性与进化》(车静等, 2020)和《湖南鱼 类原色图谱》(廖伏初等, 2020)中, 占15.6\%, 剩余 84.4\%的物种则发表于 20 种期刊。期刊论文共有 73 篇, 其中69篇以英文发表, 占论文总数的 $94.5 \%$; 涉 及17种英文期刊, 其中发表论文数量最多的6种期 刊分别为ZooKeys (23 篇)、Zootaxa (13 篇)、 Zoological Research (7篇)、Asian Herpetological Research (6篇)、Journal of Fish Biology (3篇)、 Russian Journal of Herpetology (3篇), 这6种期刊论 文数量占比达 $75.3 \%$, 而这些期刊全部为SCI期刊。 只有4篇在《兽类学报》等中文期刊发表。这表明, 中 国主办的Asian Herpetological Research和Zoological Research等英文期刊和《兽类学报》等中文期刊发 表的相关文章数量有待提高。

致谢: 资料收集和论文成稿过程中得到程继龙、葛 德燕、夏霖和彭兴文的帮助，在此一并致谢。

\section{ORCID}

王斌 (D) https://orcid.org/0000-0001-6036-5579

蔡波 (D) https://orcid.org/0000-0002-4214-9060

陈蔚涛 (D) https://orcid.org/0000-0002-4324-1926

温知新 (1D) https://orcid.org/0000-0002-8300-4845

张德志 (D) https://orcid.org/0000-0003-1523-3168

何舜平 (D) https://orcid.org/0000-0001-9087-7890

雷富民 (D) https://orcid.org/0000-0001-9920-8167

杨奇森 (D) https://orcid.org/0000-0001-9843-2378

江建平 (D) https://orcid.org/0000-0002-1051-7797

\section{参考文献}

Che J, Jiang K, Yan F, Zhang YP (2020) Amphibians and Reptiles in Tibet: Diversity and Evolution. Science Press, Beijing. (in Chinese) [车静, 蒋珂, 颜芳, 张亚平 (2020) 西藏两栖爬行动物: 多样性与进化. 科学出版社, 北京.]

Frost DR (2021) Amphibian Species of the World: An Online Reference. Version 6.1. https://amphibiansoftheworld.amnh. org/index.php. (accessed on 2021-03-30)

Gill F, Donsker D, Rasmussen P (2021) IOC World Bird List (v11.1). doi: 10.14344/IOC.ML.11.1.

Jiang ZG, Liu SY, Wu Y, Jiang XL, Zhou KY (2017) China's mammal diversity (2nd edition). Biodiversity Science, 25, 886-895. (in Chinese with English abstract) [蒋志刚, 刘少 英, 吴毅, 蒋学龙, 周开亚 (2017) 中国哺乳动物多样性 (第2版). 生物多样性, 25, 886-895.]

Liao FC, Li H, Yang X, Yuan XP, Jiang GM, Liang ZQ, Wu YA (2020) Colored Atlas of Fishes in Hunan. Science Press, Beijing. (in Chinese) [廖伏初, 李鸿, 杨金金, 袁希平, 蒋国 明, 梁志强, 伍远安 (2020) 湖南鱼类原色图谱. 科学出 版社, 北京.]

Ma KP (2015) Species Catalogue of China: A remarkable 
achievement in the field of biodiversity science in China. Biodiversity Science, 23, 137-138. (in Chinese) [马克平 (2015) 中国生物多样性编目取得重要进展. 生物多样性, 23, 137-138.]

The Biodiversity Committee of Chinese Academy of Sciences (2021) Catalogue of Life China: 2021 Annual Checklist. http://sp2000.org.cn/info/info_how_to_cite. (accessed on 2021-07-31)

Uetz P, Freed P, Aguilar R, Hošek J (2021) The Reptile Database. http://www.reptile-database.org. (accessed on 2021-07-31)

Wang K, Ren JL, Chen HM, Lü ZT, Guo XG, Jiang K, Chen
JM, Li JT, Guo P, Wang YY, Che J (2020) The updated checklists of amphibians and reptiles of China. Biodiversity Science, 28, 189-218. (in Chinese with English abstract) [王 剀, 任金龙, 陈宏满, 吕植桐, 郭宪光, 蒋珂, 陈进民, 李 家堂, 郭鹏, 王英永, 车静 (2020) 中国两栖、爬行动物 更新名录. 生物多样性, 28, 189-218.]

Zheng GM (2017) A Checklist on the Classification and Distribution of the Birds of China, 3rd edn. Science Press, Beijing. (in Chinese) [郑光美 (2017) 中国鸟类分类与分 布名录(第三版). 科学出版社, 北京.]

(责任编辑：间文杰)

\section{附录 Supplementary Material}

\section{附录12020年中国新增脊椎动物物种信息}

Appendix 1 The newly recorded vertebrate species of China in 2020

https://www.biodiversity-science.net/fileup/PDF/2021214-1.xlsx

附录2 2020年中国脊椎动物各分类阶元新增物种数

Appendix 2 Species number newly recorded in each genus, family, order, and class of vertebrate in China in 2020 https://www.biodiversity-science.net/fileup/PDF/2021214-2.xlsx

附录3 2020年中国新增脊椎动物物种的发表单位信息

Appendix 3 The institutions involved in the descriptions of newly recorded species of vertebrate in China in 2020 https://www.biodiversity-science.net/fileup/PDF/2021214-3.xlsx

附录4 2020年中国新增脊椎动物物种的发表作者信息

Appendix 4 The authors contribute to the newly recorded species of vertebrate in China in 2020 https://www.biodiversity-science.net/fileup/PDF/2021214-4.xlsx 